\title{
Potential IncRNA diagnostic biomarkers for early gastric cancer
}

\author{
QIN LU, TING YU, XILONG OU, DAZHONG CAO, TING XIE and XIA CHEN \\ Department of Gastroenterology, Zhongda Hospital, School of Medicine, \\ Southeast University, Nanjing, Jiangsu 210009, P.R. China
}

Received June 24, 2016; Accepted April 19, 2017

DOI: $10.3892 / \mathrm{mmr} .2017 .7770$

\begin{abstract}
Long noncoding RNAs (lncRNAs) serve important functions in many crucial biological processes; however, the effects of lncRNAs in early gastric cancer (EGC) are not entirely clear. The present study aimed to demonstrate the potential of lncRNAs to be used as biomarkers in EGC. Reverse transcription-quantitative polymerase chain reaction was used to measure the expression levels of lncRNAs, including $\mathrm{X}$ inactive-specific transcript (XIST), Yiya, brain cytoplasmic RNA 1 (BCYRN1), ribosomal RNA processing 1B (RRP1B), KCNQ1 opposite transcript 1 (KCNQ1OT1) and testes development related 1 (TDRG1), in EGC tissues compared with normal adjacent tissues (NATs). XIST, BCYRN1, RRP1B and TDRG1 were identified as differentially expressed in EGC tissues compared with NATs. The specificity and sensitivity of XIST, BCYRN1, RRP1B and TDRG1 were determined by receiver operating characteristic curve analysis. In addition, RRP1B expression was revealed to be significantly correlated with distal metastasis $(\mathrm{P}=0.020)$ and tumor-node-metastasis staging $(\mathrm{P}=0.018)$, and TDRG1 expression was significantly correlated with lymph node metastasis $(\mathrm{P}=0.001)$. Furthermore, BCYRN1, RRP1B and TDRG1 expression levels were compared between EGC tissues and plasma, and the results indicated that there were significant positive correlations of XIST, BCYRN1, RRP1B and TDRG1 expression levels between the EGC tissues and plasma. Therefore, the present study suggested that XIST, BCYRN1, RRP1B and TDRG1 may be served as potential diagnostic biomarkers for EGC.
\end{abstract}

\section{Introduction}

Gastric cancer (GC) is a high-incidence disease worldwide, particularly in Eastern Asia, although there has been a recent downward trend in morbidity (1); GC is the third major cause of cancer-related mortality in the world (2). Patients with early GC (EGC) may be cured completely though effective

Correspondence to: Dr Qin Lu, Department of Gastroenterology, Zhongda Hospital, School of Medicine, Southeast University, 87 Dingjiaqiao Road, Nanjing, Jiangsu 210009, P.R. China

E-mail: luqin81287@qq.com

Key words: long noncoding RNAs, biomarkers, early gastric cancer treatment. At present, although surgery, chemotherapy and radiotherapy are used in treatments, the prognosis of patients with GC is still very poor owing to metastasis (3-6). Therefore, early diagnosis serves an important role in reducing GC-related mortality. However, as there are no effective diagnostic signs or sensitive biomarkers for early diagnosis, most GC patients develop terminal cancer (7). Therefore, identifying specific biomarkers and effective molecular targets for GC are extremely important.

Long noncoding RNAs (lncRNAs) are a type of noncoding RNA that are $>200$ nucleotides, which regulate gene expression through transcription regulation, post-transcription regulation, chromatin modification and genomic imprinting $(8,9)$. An increasing number of studies have indicated that lncRNAs participated in various biological processes, such as cell cycle and cell differentiation (10), apoptosis $(11,12)$, epithelial-mesenchymal transition (EMT), cell migration and metastasis (13). Several previous studies have reported that IncRNAs may be closely associated with tumor genesis, including liver cancer (14), lung cancer (15), ovarian cancer (16), colorectal cancer (17) and breast cancer (18-20). Therefore, IncRNAs may be potential diagnostic biomarkers for certain diseases. A previous report using a human lncRNA microarray identified 33 differentially expressed lncRNAs associated with EGC, including 13 that were upregulated and 20 downregulated (21). The present study further validated that $\mathrm{X}$ inactive-specific transcript (XIST), brain cytoplasmic RNA 1 (BCYRN1), ribosomal RNA processing 1B (RRP1B) and testes development related 1 (TDRG1) were aberrantly expressed both in EGC tissues and plasma.

The present study examined the expression levels of XIST, Yiya, BCYRN1, RRP1B, KCNQ1 opposite transcript 1 (KCNQ1OT1) and TDRG1 in EGC tissues and normal adjacent tissues (NATs) by reverse transcription-quantitative polymerase chain reaction (RT-qPCR). XIST, BCYRN1, RRP1B and TDRG1 were identified as differentially expressed in EGC tissues compared with NATs, and exhibited potential diagnostic values for the detection of EGC. The expression level of RRP1B was significantly correlated with distal metastasis and tumor-node-metastasis (TNM) staging, and the expression of TDRG1 was significantly correlated with lymph node metastasis. Furthermore, significant positive correlations for XIST, BCYRN1, RRP1B and TDRG1 expression levels were made between the EGC tissues and plasma. Therefore, XIST, BCYRN1, RRP1B and TDRG1 may serve as potential diagnostic biomarkers for EGC. 


\section{Materials and methods}

Clinical specimens. The present study was approved by the Ethics committee of Zhongda Hospital, School of Medicine, Southeast University (Nanjing, China), and informed consent was received from each patient. A total of 76 pairs of EGC tissues and paired NATs were collected from Zhongda Hospital between May 2014 and November 2016. Among them, 10 pairs of EGC tissues and paired NATs were used to preliminarily detect the XIST, Yiya, BCYRN1, RRP1B, KCNQ1OT1 and TDRG1 expression levels. The 10 patients included 5 males and 5 female patients, and the average age was 63.4 and 64.2 years old. None of the patients in the study received radiotherapy or chemotherapy prior to surgical resection. All collected tissue samples were frozen at $-80^{\circ} \mathrm{C}$ for total RNA extraction. Peripheral blood $(5 \mathrm{ml})$ was collected from the 76 fasting patients prior to endoscopy, and controls ( 76 healthy patients, including 32 males and 44 female patients, and the average age was 65.4 and 61.2 years old) were done at the same time; serum was separated by centrifugation $(3,500 \mathrm{x} \mathrm{g}$; $10 \mathrm{~min} ; 10^{\circ} \mathrm{C}$ ) and the serum supernatant was frozen at $-80^{\circ} \mathrm{C}$ until further analysis.

$R T-q P C R$. Total RNA was extracted from EGC tissues (100 mg for every organization) and paired NATs using the RNeasy Plus Mini kit (Qiagen, Inc., Valencia, CA, USA) according to the manufacturer's protocol. Total RNA was extracted from serum $(800 \mu \mathrm{l})$ using the QIAamp Circulating Nucleic Acid kit (QiagenKK, Tokyo, Japan). RNA purity was measured using the NanoDrop (Peqlab Biotechnologie $\mathrm{GmbH}$, Erlangen, Germany). The $\mathrm{OD}_{260 / 280}$ ratio was used as indicator for RNA purity. A ratio higher than 1.8 was regarded as suitable for gene expression measurements. The RevertAid First Strand cDNA Synthesis kit (Thermo Fisher Scientific, Inc., Waltham, MA, USA) was used to synthesize cDNA according to the manufacturer's protocol. XIST, Yiya, BCYRN1, RRP1B, KCNQ1OT1 and TDRG1 expression were examined in EGC tissues (paired NATs were used as control) and EGC plasma (healthy patient serum were used as control) by RT-qPCR using the SYBRGreen Master Mix kit (Takara Bio, Inc., Otsu Japan) and PRISM 7900HT sequence detection system (Applied Biosystems; Thermo Fisher Scientific, Inc.) as described previously (22). The quantitative PCRs were carried out in $20-\mu 1$ reaction volume containing $2 \mu 1 \mathrm{cDNA}$ products. Reaction steps were as follows: $95^{\circ} \mathrm{C}$ for $30 \mathrm{sec}$ (predegeneration) as the first step in a loop; $95^{\circ} \mathrm{C}$ for $5 \mathrm{sec}$ (degeneration), $60^{\circ} \mathrm{C}$ for $34 \mathrm{sec}$ (extension) as the second step, a total of 40 cycles. The data was analyzed using SDS 2.3 software (Applied Biosystems; Thermo Fisher Scientific, Inc.). The specificity of primer sequences was measured according to the dissociation curve, and the relative gene expression levels were analyzed using the $2^{-\Delta \Delta \mathrm{Cq}}$ (quantitation threshold) method (23). All data are presented as the mean \pm standard deviation of three independent experiments. The following qPCR primers were used: XIST, forward 5'-AACCAC CTACACTTGAGCCA-3', reverse 5'-AGGACAATGACG AAGCCACT-3'; Yiya, forward 5'-TTGAGTCGGATCCTC TCAGC-3', reverse 5'-CTCTCTGAGTTGCCCTTGGA-3'; BCYRN1, forward 5'-TCATGAAGCTTGCCTCTGGA-3', reverse 5'-AACATGGAGAGGGAAGGTGG-3'; RRP1B, forward 5'-CACAGCACAAACACGAGTCA-3', reverse 5'-TGCCTTCTACTTGGTGAGGG-3'; KCNQ1OT1, forward 5'-TGGTAAGTTACAGGGCAGGG-3', reverse 5'-TGAACA TCCATCCCCAAGCT-3'; TDRG1, forward 5'-GGTGCAGTC TTCAGGGATCT-3', reverse 5'-GCCTCCCTCCTCTTCATT GT-3'; GAPDH, forward 5'-TGTTCGTCATGGGTGTGA AC-3', reverse 5'-ATGGCATGGACTGTGGTCAT-3'. Samples were normalized to GAPDH.

Statistical analysis. All data and calculations were analyzed using Prism6 (GraphPad Software Inc., La Jolla, CA, USA) and SPSS 17.0 (SPSS, Inc., Chicago, IL, USA). The area under the receiver operating characteristic (ROC) curve (AUC) was used to assess the predictive power and to determine the cut-off scores for XIST, BCYRN1, RRP1B and TDRG1 expression levels between patients with EGC and controls. The differences in lncRNA expressions (XIST, BCYRN1, RRP1B and TDRG1) in tissues among the patients were analyzed using the $\chi^{2}$ test concerning clinical parameters such as age ( $>60$ vs. $<60$ years), sex (male vs. female), pathological node (pN status; N0 vs. N1-N2), pathological metastasis (pM status; M0 vs. M1), and clinical stage (I and II vs. III and IV). For paracarcinoma-carcinoma paired tissues, the difference in IncRNA expression was evaluated using paired Student's t-test. The relationship of lncRNA expression in EGC tissue and plasma was analyzed using Mantel-Haenszel statistics. All results are presented as the mean \pm standard deviation (SD). $\mathrm{P}<0.05$ was considered to indicate a statistically significant difference.

\section{Results}

XIST, Yiya, BCYRN1, RRPIB, KCNQ1OT1 and TDRG1 expression levels in EGC tissues and NATs. A previous study analyzed IncRNA expression profiles including XIST, Yiya, BCYRN1, RRP1B, KCNQ1OT1 and TDRG1 in GC tissues and paired NATs by a human IncRNA microarray (21). This previous study identified 68 lncRNAs that were associated with diseases, of which the top 33 were demonstrated to be differentially expressed, including 13 upregulated and 20 downregulated lncRNAs. As hypoxia inducible factor $1 \alpha$-antisense RNA 1 (HIF1 $\alpha-A S 1)$, plasmacytoma variant translocation 1 (PVT1), carbonyl reductase 3-antisense RNA 1 (CBR3-AS1) and urothelial cancer associated 1 (UCA1) have been identified previously, the present study further examined the expression levels of XIST, Yiya, BCYRN1, RRP1B, KCNQ1OT1 and TDRG1 by RT-qPCR in EGC tissues and NATs (21). Initially, we identified six abnormally expressed lncRNAs in 10 tissues as a preliminary screening. The results demonstrate that the expression levels of XIST (Fig. 1A) and BCYRN1 (Fig. 1B) were significantly increased in EGC tissues compared with NATs $\left(\mathrm{n}=10 ;{ }^{* *} \mathrm{P}<0.01\right)$; Yiya (Fig. $\left.1 \mathrm{C}\right)$ and KCNQ1OT1 (Fig. 1D) exhibited no significant changes in expression levels in EGC tissues compared with NATs $(\mathrm{n}=10)$; and the expression levels of RRP1B (Fig. 1E) and TDRG1 (Fig. 1F) were significantly decreased in EGC tissues compared with NATs $(n=10$; ${ }^{*} \mathrm{P}<0.05$ and ${ }^{* *} \mathrm{P}<0.01$, respectively).

XIST, BCYRN1, RRPIB and TDRG1 expression levels were validated in EGC tissues and NATs. In the preliminary study 
A

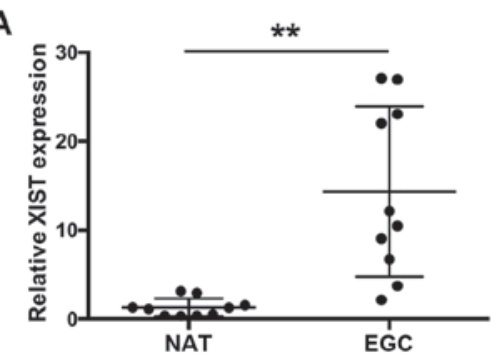

D

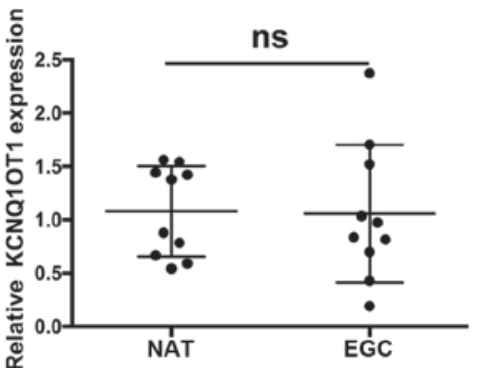

B

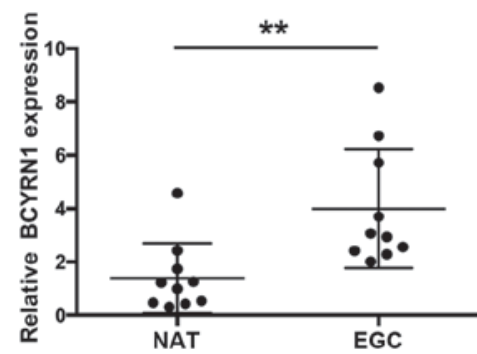

E

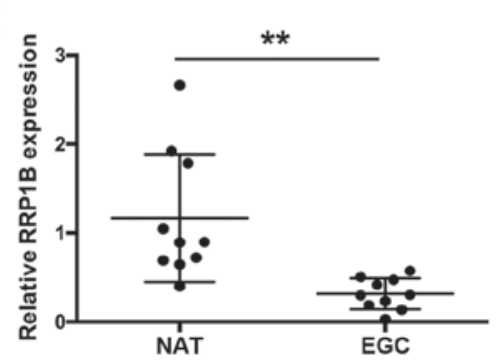

C

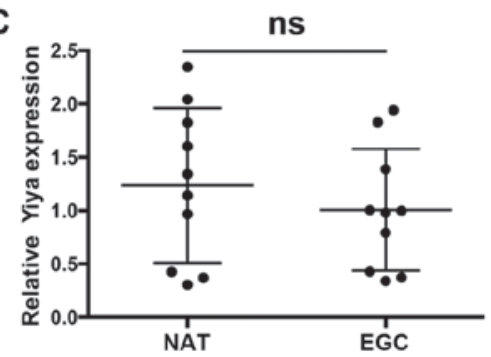

F

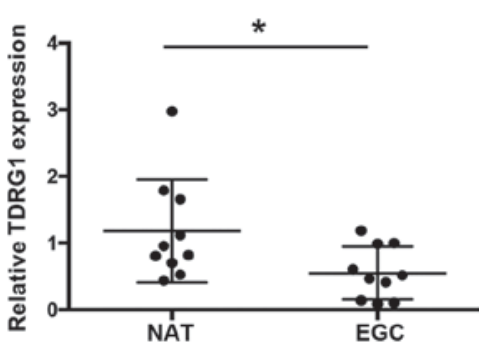

Figure 1. XIST, Yiya, BCYRN1, RRP1B, KCNQ1OT1 and TDRG1 expression levels in EGC tissues and NATs. Reverse transcription-quantitative polymerase chain reaction was used to detect the expression levels of (A) XIST, (B) BCYRN1, (C) Yiya, (D) KCNQ1OT1, (E) RRP1Band (F) TDRG1 in EGC tissues and paired NATs. Relative expression levels were normalized to GAPDH using the $2^{-\Delta \Delta C q}$ method. $\mathrm{n}=10 ;{ }^{*} \mathrm{P}<0.05 ;{ }^{* * *} \mathrm{P}<0.01$. BCYRN1, brain cytoplasmic RNA 1; EGC, early gastric cancer; KCNQ1OT1, KCNQ1 opposite transcript 1; NAT, normal adjacent tissue; ns, no statistically significant difference; RRP1B, ribosomal RNA processing 1B; TDRG1, testes development related 1; XIST, X inactive-specific transcript.

A

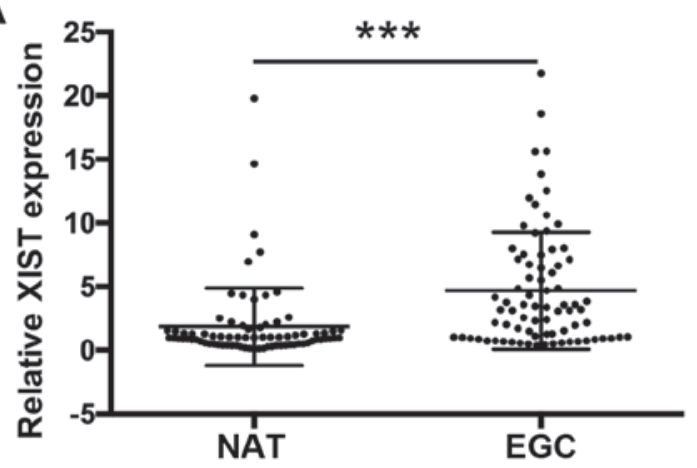

C

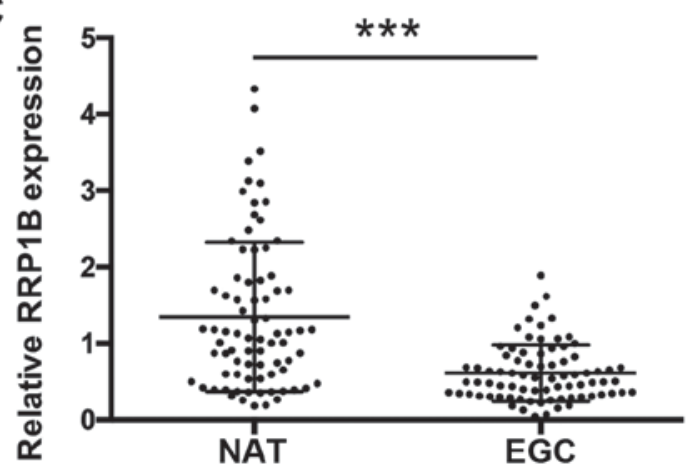

B

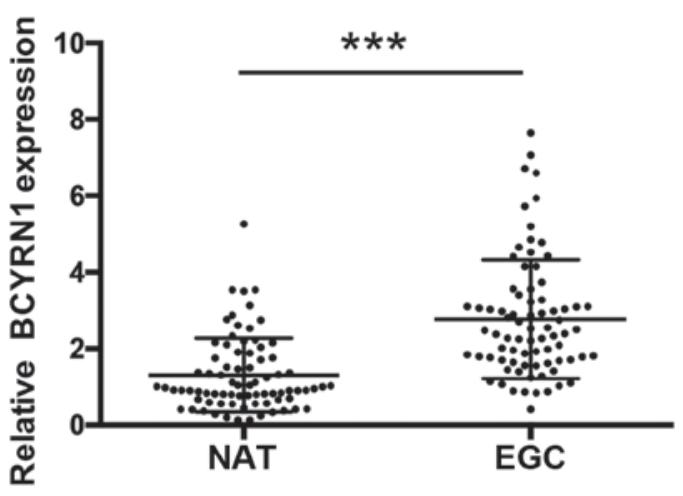

D

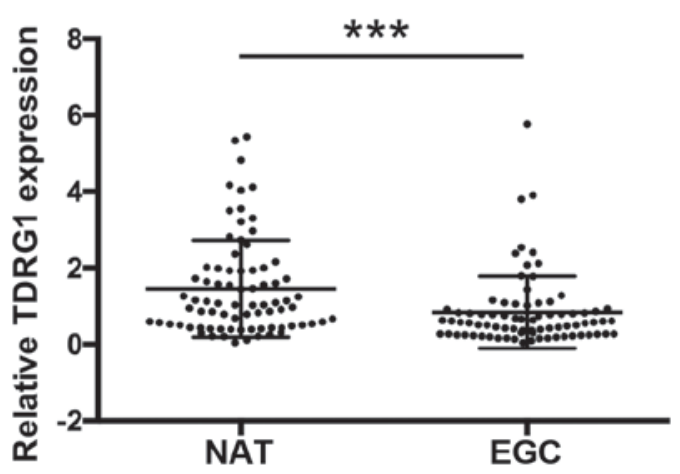

Figure 2. XIST, BCYRN1, RRP1B and TDRG1 expression levels in EGC tissues and NATs. Reverse transcription-quantitative polymerase chain reaction was used to detect the expression levels of (A) XIST, (B) BCYRN1, (C) RRP1B and (D) TDRG1 in EGC tissues compared with paired NATs from 78 patients. Relative expression levels were normalized to GAPDH using the $2^{-\triangle \Delta C q}$ method. ${ }^{* * *} \mathrm{P}<0.001$. BCYRN1, brain cytoplasmic RNA 1; EGC, early gastric cancer; NAT, normal adjacent tissue; RRP1B, ribosomal RNA processing 1B; TDRG1, testes development related 1; XIST, X inactive-specific transcript.

described above, XIST, BCYRN1, RRP1B and TDRG1 were differentially expressed in EGC tissues ( $\mathrm{n}=10)$ compared with NATs ( $\mathrm{n}=10)$, while Yiya and KCNQ1OT1 expressions exhibited no significant alterations in EGC tissues compared with NATs. Therefore, we further collected the EGC tissues and paired NATs from 76 patients. The expression levels of XIST, BCYRN1, RRP1B and TDRG1 were measured by RT-qPCR. The results demonstrated that the expression levels of XIST (Fig. 2A) and BCYRN1 (Fig. 2B) were significantly increased in EGC tissues compared with NATs $\left(n=76 ;{ }^{* * *} \mathrm{P}<0.001\right)$. 
Table I. Associations between XIST expression level and clinicopathological characteristics in 76 patients.

\begin{tabular}{|c|c|c|c|c|}
\hline \multirow{2}{*}{$\begin{array}{l}\text { Clinicopathological } \\
\text { characteristic }\end{array}$} & \multirow[b]{2}{*}{$\mathrm{n}$} & \multicolumn{3}{|c|}{ XIST } \\
\hline & & High expression (\%) & Low expression (\%) & P-value \\
\hline \multicolumn{5}{|l|}{ Age (year) } \\
\hline$>60$ & 47 & $42(51.1)$ & $23(48.9)$ & \multirow[t]{2}{*}{0.097} \\
\hline$\leq 60$ & 29 & $20(69.0)$ & $9(31.0)$ & \\
\hline \multicolumn{5}{|l|}{ Sex } \\
\hline Male & 50 & $29(58.0)$ & $21(42.0)$ & \multirow[t]{2}{*}{0.585} \\
\hline Female & 26 & $15(57.7)$ & $11(42.3)$ & \\
\hline \multicolumn{5}{|l|}{ pN status } \\
\hline N0 & 44 & $29(65.9)$ & $15(34.1)$ & \multirow[t]{2}{*}{0.077} \\
\hline N1-N2 & 32 & 15 (46.9) & $17(53.1)$ & \\
\hline \multicolumn{5}{|l|}{ pM status } \\
\hline M0 & 71 & $41(57.7)$ & $30(42.3)$ & \multirow[t]{2}{*}{0.649} \\
\hline M1 & 5 & $3(60.0)$ & $2(40.0)$ & \\
\hline \multicolumn{5}{|l|}{ Clinical stage } \\
\hline I and II & 46 & 28 (60.9) & $18(39.1)$ & \multirow[t]{2}{*}{0.339} \\
\hline III and IV & 30 & $16(53.3)$ & $14(46.7)$ & \\
\hline
\end{tabular}

The P-values of XIST expressions (high expression vs. low expression) were $0.097,0.585,0.077,0.649$ and 0.339 compared with age (>60 vs. $<60$ vs.), sex (Male vs. Female), pN status (N0 vs. N1-N2), pM status (M0 vs. M1), and clinical stage (I and II vs. III and IV). pM, pathological metastasis; pN, pathological node; XIST, X inactive-specific transcript. The differences of XIST expression in tissues among the patients was analyzed using a $\chi^{2}$ test concerning clinical parameters.

In addition, the expression levels of RRP1B (Fig. 2C) and TDRG1 (Fig. 2D) were significantly decreased in EGC tissues compared with NATs $\left(\mathrm{n}=76 ;{ }^{* * *} \mathrm{P}<0.001\right)$. Therefore, XIST, BCYRN1, RRP1B and TDRG1may serve as potential candidates as biomarkers for EGC.

In addition, no significant correlations were identified between the expression levels of XIST or BCYRN1 and clinicopathological characteristics (Tables I and II, respectively). Conversely, the expression level of RRP1B was significantly correlated with pathological metastasis (pM) and clinical stage (Table III), and the expression level of TDRG1 was significantly correlated with pathological node (pN) (Table IV).

XIST, BCYRN1, RRPIB and TDRG1 may be used as noninvasive biomarkers for EGC. The ROC curve is a comprehensive index that reflects the sensitivity and specificity of continuous variables. In the present study, the occurrence of EGC was predicted by ROC curve analysis using XIST, BCYRN1, RRP1B and TDRG1 expressions in 76 EGC samples and paired NATs (controls). The AUC for XIST was 0.733 (sensitivity=0.846; specificity $=0.590 ;{ }^{* * *} \mathrm{P}<0.001$; Fig. 3A). The AUC for BCYRN1 was 0.821 (sensitivity $=0.679$; specificity $=0.859 ;{ }^{* * *} \mathrm{P}<0.001$; Fig. 3B). The AUC for RRP1B was 0.753 (sensitivity=0.859; specificity $=0.564 ;{ }^{* * *} \mathrm{P}<0.001$; Fig. $\left.3 \mathrm{C}\right)$. The AUC for TDRG1 was 0.681 (sensitivity $=0.731$; specificity $=0.603$; ${ }^{* * *} \mathrm{P}<0.001$; Fig. 3D). These data suggested that XIST, BCYRN1, RRP1B and TDRG1 may be able to serve as biomarkers of EGC.
Positive correlation of XIST, BCYRN1, RRPIB and TDRG1 expression between EGC tissue and plasma. Similar to EGC tissues, the expression levels of XIST and BCYRN1 were increased and the expression levels of RRP1B and TDRG1 were decreased in the plasma from patients with EGC. The correlations for XIST, BCYRN1, RRP1B and TDRG1 expression levels between EGC tissue and plasma were further analyzed and the results indicated that there was a positive correlation for XIST expression between EGC tissues and plasma $\left(\mathrm{R}^{2}=0.2650 ;{ }^{* * *} \mathrm{P}<0.001\right.$; Fig. 4A). A positive correlation was also made between EGC tissues and plasma for BCYRN1 expression $\left(\mathrm{R}^{2}=0.2686 ;^{* * *} \mathrm{P}<0.001\right.$; Fig. 4B), RRP1B expression $\left(\mathrm{R}^{2}=0.2920 ;{ }^{* * *} \mathrm{P}<0.001\right.$; Fig. 4C), and TDRG1 expression $\left(\mathrm{R}^{2}=0.4120 ;{ }^{* * *} \mathrm{P}<0.001\right.$; Fig. 4D). These results demonstrated that XIST, BCYRN1, RRP1B and TDRG1 were aberrantly expressed both in EGC tissues and plasma, which may be related to EGC disease progression.

\section{Discussion}

Currently, the study of biomarkers study has focused on noncoding RNAs, particularly lncRNAs, most of which are transcribed by RNA polymerase (Pol) II and Pol I, but some are transcribed by RNA Pol III (24). A number of previous studies have indicated that lncRNAs serve important roles in regulating gene expression (20,25-27) and participate in cell cycle and differentiation $(10)$, apoptosis $(11,12)$ and chromatin remodeling (28-30). Other studies have demonstrated that IncRNAs were involved in the development of various 
Table II. Associations between BCYRN expression level and clinicopathological characteristics in 76 patients.

Clinicopathological

characteristic

$\mathrm{n}$

\section{BCYRN1}

$\begin{array}{lccc}\begin{array}{l}\text { Age (year) } \\ >60\end{array} & 47 & 41(87.2) & 6(12.8) \\ \leq 60 & 29 & 24(82.8) & 5(17.2) \\ \text { Sex } & & & 6(12.0) \\ \text { Male } & 50 & 44(88.0) & 5(19.2) \\ \text { Female } & 26 & 21(80.8) & 7(15.9) \\ \text { pN status } & & & 4(12.5) \\ \text { N0 } & 44 & 37(84.1) & 11(15.5) \\ \text { N1-N2 } & 32 & 28(87.5) & 0(0.0) \\ \text { pM status } & & 60(84.5) & 0.471 \\ \text { M0 } & 71 & 5(100.0) & 6(13.0) \\ \text { M1 } & 5 & & 5(16.7) \\ \text { Clinical stage } & & 40(87.0) & 0.447 \\ \text { I and II } & 46 & 25(83.3) & 0.452 \\ \text { III and IV } & 30 & & \end{array}$

None of the P-values in the Table indicates $<0.05$. The P-values of BCYRN1 expressions (high expression vs. low expression) were 0.413, $0.300,0.471,0.447$ and 0.452 compared with age (>60 vs. $<60$ vs.), sex (Male vs. Female), pN status (N0 vs. N1-N2), pM status (M0 vs. M1), and clinical stage (I and II vs. III and IV). BCYRN1, brain cytoplasmic RNA 1; pM, pathological metastasis; pN, pathological node. The differences of BCYRN1 expression in tissues among the patients was analyzed using a $\chi^{2}$ test concerning clinical parameters.

Table III. Associations between RRP1B expression level and clinicopathological characteristics in 76 patients.

\begin{tabular}{|c|c|c|c|c|}
\hline \multirow{2}{*}{$\begin{array}{l}\text { Clinicopathological } \\
\text { characteristic }\end{array}$} & \multirow[b]{2}{*}{$\mathrm{n}$} & \multicolumn{3}{|c|}{ RRP1B } \\
\hline & & High expression (\%) & Low expression $(\%)$ & P-value \\
\hline \multicolumn{5}{|l|}{ Age (year) } \\
\hline$>60$ & 47 & 7 (14.9) & $40(85.1)$ & 0.587 \\
\hline$\leq 60$ & 29 & $4(13.8)$ & $25(86.2)$ & \\
\hline \multicolumn{5}{|l|}{ Sex } \\
\hline Male & 50 & $7(14.0)$ & $43(86.0)$ & 0.561 \\
\hline Female & 26 & $4(15.4)$ & $22(84.6)$ & \\
\hline \multicolumn{5}{|l|}{ pN status } \\
\hline No & 44 & 7 (15.9) & $37(84.1)$ & 0.471 \\
\hline N1-N2 & 32 & $4(12.5)$ & $28(87.5)$ & \\
\hline \multicolumn{5}{|l|}{ pM status } \\
\hline M0 & 71 & $8(11.3)$ & $63(88.7)$ & $0.020^{\mathrm{a}}$ \\
\hline M1 & 5 & $3(60.0)$ & $2(40.0)$ & \\
\hline \multicolumn{5}{|l|}{ Clinical stage } \\
\hline I and II & 46 & $8(6.5)$ & $43(93.5)$ & $0.018^{\mathrm{a}}$ \\
\hline III and IV & 30 & $3(26.7)$ & $22(73.3)$ & \\
\hline
\end{tabular}

None of the P-values in the Table indicates $<0.05 . \mathrm{P}<0.05$ was considered to indicate a statistically significant difference. The $\mathrm{P}$-values of RRP1B expressions (high expression vs. low expression) were $0.587,0.561,0.471,0.020$ and 0.018 compared with age ( $>60$ vs. $<60$ vs.), sex (Male vs. Female), pN status (N0 vs. N1-N2), pM status (M0 vs. M1), and clinical stage (I and II vs. III and IV). ${ }^{\mathrm{a}} \mathrm{P}<0.05$. pM, pathological metastasis; pN, pathological node; RRP1B, ribosomal RNA processing 1B. The differences of RRP1B expression in tissues among the patients was analyzed using a $\chi^{2}$ test concerning clinical parameters. 
Table IV. Associations between TDRG1 expression level and clinicopathological characteristics in 76 patients.

TDRG1

\begin{tabular}{|c|c|c|c|c|}
\hline \multirow{2}{*}{$\begin{array}{l}\text { Clinicopathological } \\
\text { characteristic }\end{array}$} & \multirow[b]{2}{*}{$\mathrm{n}$} & \\
\hline & & High expression (\%) & Low expression $(\%)$ & P-value \\
\hline \multicolumn{5}{|l|}{ Age (year) } \\
\hline$>60$ & 47 & $16(34.0)$ & $31(66.0)$ & 0.162 \\
\hline$\leq 60$ & 29 & $6(20.7)$ & $23(79.3)$ & \\
\hline \multicolumn{5}{|l|}{ Sex } \\
\hline Male & 50 & $17(34.0)$ & $33(66.0)$ & 0.140 \\
\hline Female & 26 & $5(19.2)$ & $21(80.8)$ & \\
\hline \multicolumn{5}{|l|}{ pN status } \\
\hline No & 44 & $19(43.2)$ & $25(26.8)$ & $0.001^{\mathrm{a}}$ \\
\hline N1-N2 & 32 & $3(9.4)$ & $29(90.6)$ & \\
\hline \multicolumn{5}{|l|}{ pM status } \\
\hline M0 & 71 & $21(29.6)$ & $50(70.4)$ & 0.548 \\
\hline M1 & 5 & $1(20.0)$ & $4(80.0)$ & \\
\hline \multicolumn{5}{|l|}{ Clinical stage } \\
\hline I and II & 46 & $11(23.9)$ & $35(76.1)$ & 0.174 \\
\hline III and IV & 30 & $11(36.7)$ & $19(63.3)$ & \\
\hline
\end{tabular}

None of the P-values in the Table indicates $<0.05 . \mathrm{P}<0.05$ was considered to indicate a statistically significant difference. The $\mathrm{P}$-values of TDRG1 expressions (high expression vs. low expression) were $0.162,0.140,0.001,0.548$ and 0.174 compared with age ( $>60$ vs. $<60$ vs.), sex (Male vs. Female), pN status (N0 vs. N1-N2), pM status (M0 vs. M1), and clinical stage (I and II vs. III and IV). ${ }^{\mathrm{a} P<0.05}$. pM, pathological metastasis; pN, pathological node; TDRG1, testes development related 1. The differences of TDRG1 expression in tissues among the patients was analyzed using a $\chi^{2}$ test concerning clinical parameters.

cancers (31). For example, long intergenic noncoding RNA for kinase activation (LINK-A) was reported to activate normoxic HIF1 $\alpha$ signaling in certain breast cancers (32); antisense noncoding RNA in the INK4 locus (ANRIL) may be a potential prognostic biomarker in GC and has been demonstrated to regulate microRNA (miR)-99a/miR-449a (33); and lncRNA-n336928 has been correlated with bladder cancer tumor stage and overall survival (34). Therefore, lncRNAs may be important regulatory factors for gene expressions, yet their functions in cancer remain unclear and requires a deeper understanding of the regulatory networks that may be involved.

A previous study identified 33 differentially expressed lncRNAs using a human lncRNA microarray to screen GC tissues and paired NATs (21). Other studies have reported that $\mathrm{H} 19$ promotes proliferation of GC cells and high expression of $\mathrm{H} 19$ indicates a poor prognosis in patients with GC $(35,36)$; prostate cancer associated 3 (PCA3) is highly expressed in prostate cancer (37); HOX transcript antisense RNA (HOTAIR) promotes tumor invasion and reverses EMT in GC $(38,39)$. In addition, a decrease in the expression of growth arrest specific 5 (GAS5) was revealed to induce a poor prognosis and accelerate cell proliferation in GC (40), and metastasis associate lung adenocarcinoma transcript 1 (MALAT1) was reported to enhance GC cell proliferation through pre-mRNA-splicing factor SF2/alternative splicing factor (ASF) (41). HIF1A-AS1, PVT1, CBR3-AS1 and UCA1 have also been identified in GC (21), and the present study examined the expression levels of XIST, Yiya, BCYRN1, RRP1B, KCNQ1OT1 and TDRG1 in EGC.

In the present study, it was demonstrated that XIST and BCYRN1 were significantly upregulated, and RRP1B and TDRG1 were significantly downregulated, in EGC tissues compared with NATs. RRP1B was correlated with pM and clinical stage, and TDRG1 was correlated with $\mathrm{pN}$. In addition, there were positive correlations for XIST, BCYRN1, RRP1B and TDRG1 expressions between EGC tissue and plasma. Therefore, it was suggested that XIST, BCYRN1, RRP1B and TDRG1 may be promising candidates for the diagnosis of EGC.

In conclusion, RT-qPCR analysis demonstrated that XIST, BCYRN1, RRP1B and TDRG1 were differentially expressed in EGC tissues compared with NATs, and ROC curve analysis indicated that these lncRNAs have potential diagnostic values for the detection of EGC. Furthermore, the results indicated that there were significant positive correlations of XIST, BCYRN1, RRP1B and TDRG1 expression levels between the EGC tissues and plasmas. Therefore, the present study suggested that XIST, BCYRN1, RRP1B and TDRG1 may potentially serve as diagnostic biomarkers for EGC.

\section{Acknowledgements}

The present study was supported by the Jiangsu Provincial Traditional Chinese Medicine Bureau of Science and Technology Project (grant no. YB2015181). 

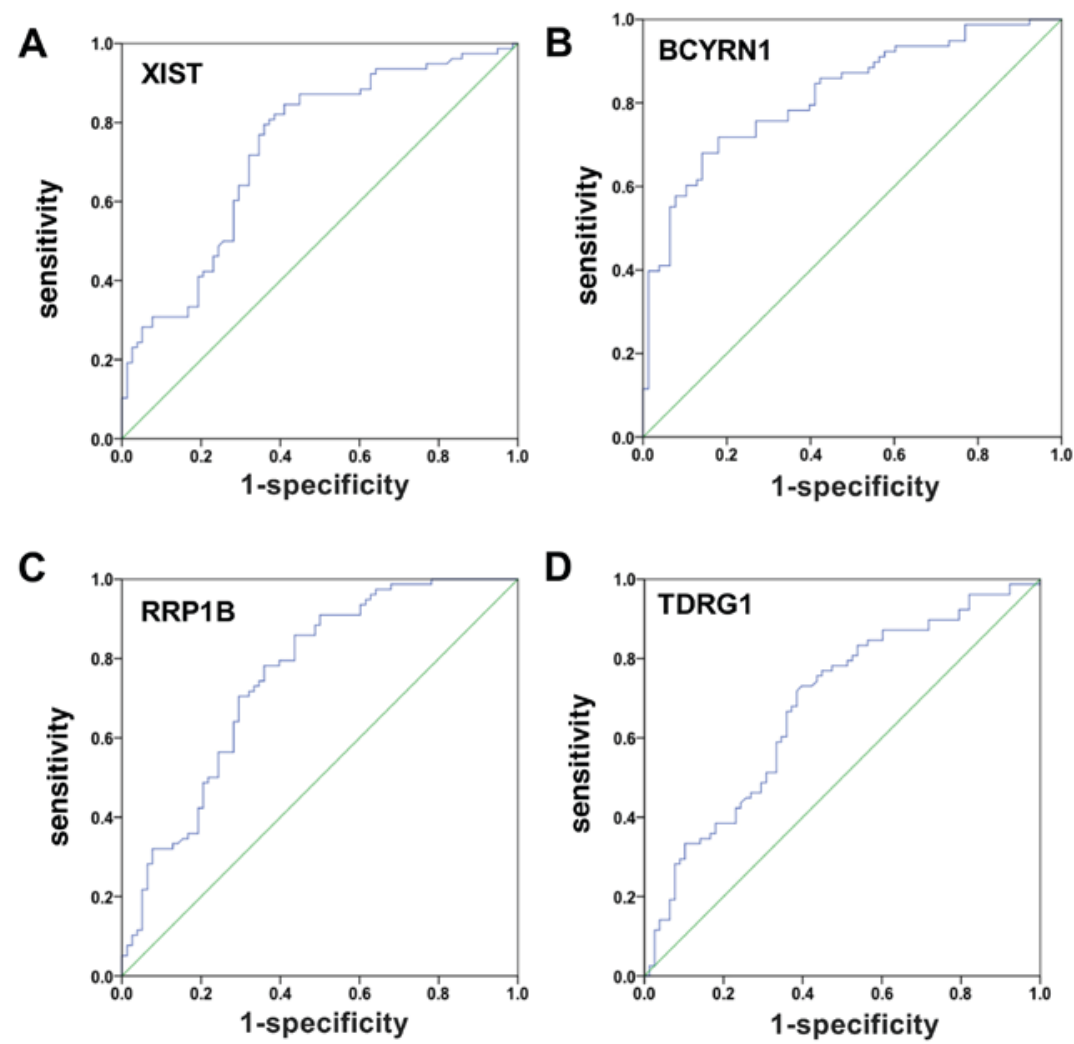

Figure 3. EGC was predicted by ROC curve analysis using XIST, BCYRN1, RRP1B and TDRG1 expression levels between EGC patients and controls. (A) ROC curve analysis using XIST.AUC $=0.733$; sensitivity $=0.846$; specificity $=0.590$. (B) ROC curve analysis using $B C Y R N 1$. AUC $=0.821$; sensitivity $=0.679$; specificity $=0.859$. (C) ROC curve analysis using RRP1B. AUC $=0.753$; sensitivity $=0.859$; specificity $=0.564$. (D) ROC curve analysis using TDRG1. AUC $=0.681$; sensitivity $=0.731$; specificity $=0.603$. An ROC curve plots the sensitivity on the $y$-axis against one minus the 1 -specificity on the $x$-axis. A diagonal line at 45 , known as the line of chance, would result from a test which allocated subjects randomly. AUC, area under the ROC curve; BCYRN1, brain cytoplasmic RNA 1; EGC, early gastric cancer; NAT, normal adjacent tissue; ROC, receiver-operator characteristic; RRP1B, ribosomal RNA processing 1B; TDRG1, testes development related 1; XIST, $\mathrm{X}$ inactive-specific transcript.

A

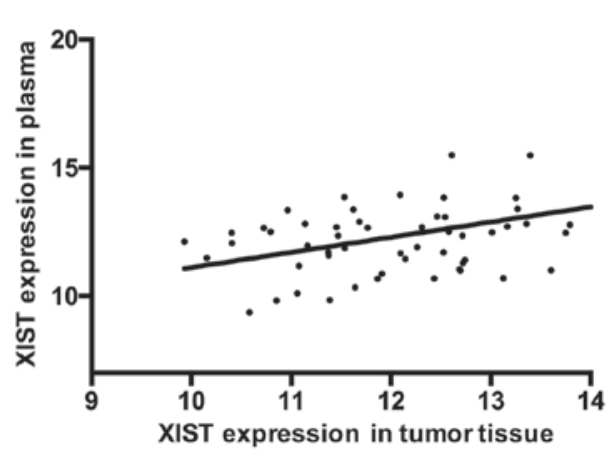

C

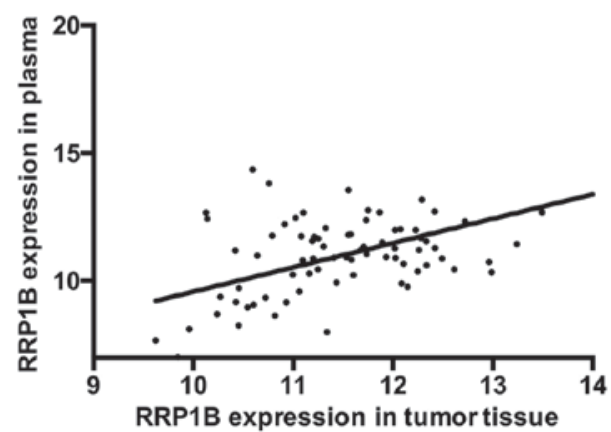

B

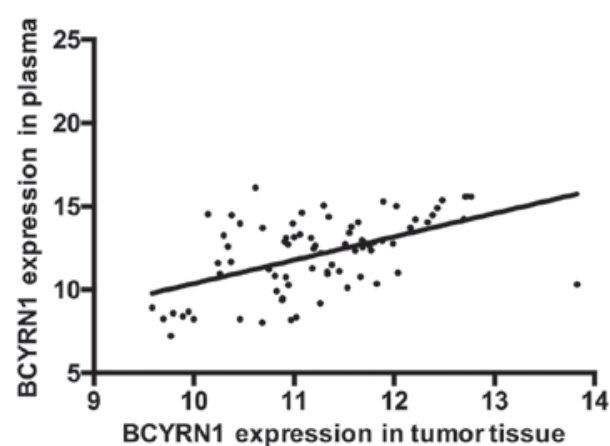

D

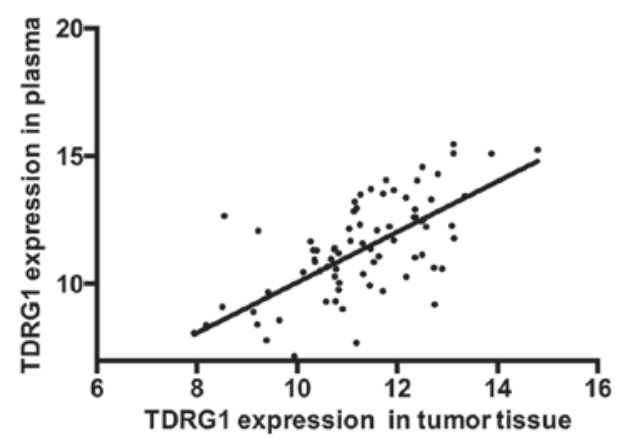

Figure 4. Correlations for XIST, BCYRN1, RRP1B and TDRG1 expression levels were determined between early gastric cancer tissue and plasma. (A) Correlation analysis of XIST. $\mathrm{R}^{2}=0.2650$. (B) Correlation analysis of $B C Y R N 1 . \mathrm{R}^{2}=0.2686$. (C) Correlation analysis of RRP1B. $\mathrm{R}^{2}=0.2920$. (D) Correlation analysis of TDRG1. $\mathrm{R}^{2}=0.4120$. BCYRN1, brain cytoplasmic RNA 1; RRP1B, ribosomal RNA processing 1B; TDRG1, testes development related 1; XIST, X inactive-specific transcript. 


\section{References}

1. Torre LA, Bray F, Siegel RL, Ferlay J, Lortet-Tieulent J and Jemal A: Global cancer statistics, 2012. CA Cancer J Clin 65 . 87-108, 2015

2. Lin M, Shi C, Lin X, Pan J, Shen S, Xu Z and Chen Q: sMicroRNA-1290 inhibits cells proliferation and migration by targeting FOXA1 in gastric cancer cells. Gene 582: 137-142, 2016.

3. Baĭramov RB and Abdullaeva RT: The impact of early gastric cancer diagnosis on indices of survival in patients after radical surgical intervention. Klin Khir: 18-21, 2013

4. Bang CS, Baik GH, Shin IS, Kim JB, Suk KT, Yoon JH, Kim YS and Kim DJ: Helicobacter pylori Eradication for prevention of metachronous recurrence after endoscopic resection of early gastric cancer. J Korean Med Sci 30: 749-756, 2015.

5. Pasechnikov V, Chukov S, Fedorov E, Kikuste I and Leja M: Gastric cancer: Prevention, screening and early diagnosis. World J Gastroenterol 20: 13842-13862, 2014.

6. Waddell T, Verheij M, Allum W, Cunningham D, Cervantes A and Arnold D; European Society for Medical Oncology (ESMO); European Society of Surgical Oncology (ESSO); European Society of Radiotherapy and Oncology (ESTRO): Gastric cancer: ESMO-ESSO-ESTRO clinical practice guidelines for diagnosis, treatment and follow-up. Eur J Surg Oncol 40 584-591, 2014.

7. Cai H, Yuan Y, Hao YF, Guo TK, Wei X and Zhang YM: Plasma microRNAs serve as novel potential biomarkers for early detection of gastric cancer. Med Oncol 30: 452, 2013.

8. Guttman M and Rinn JL: Modular regulatory principles of large non-coding RNAs. Nature 482: 339-346, 2012.

9. Khalil AM, Guttman M, Huarte M, Garber M, Raj A, Rivea Morales D, Thomas K, Presser A, Bernstein BE, van Oudenaarden A, et al: Many human large intergenic noncoding RNAs associate with chromatin-modifying complexes and affect gene expression. Proc Natl Acad Sci USA 106: 11667-11672, 2009.

10. Liu X, Li D, Zhang W, Guo M and Zhan Q: Long non-coding RNA gadd7 interacts with TDP-43 and regulates Cdk6 mRNA decay. EMBO J 31: 4415-4427, 2012.

11. Lakhotia SC: Long non-coding RNAs coordinate cellular responses to stress. Wiley Interdiscip Rev RNA 3: 779-796, 2012.

12. Paralkar VR and Weiss MJ: A new 'Linc' between noncoding RNAs and blood development. Genes Dev 25: 2555-2558, 2011.

13. Dhamija S and Diederichs S: From junk to master regulators of invasion: lncRNA functions in migration, EMT and metastasis. Int J Cancer 139: 269-280, 2016.

14. Yang F, Zhang L, Huo XS, Yuan JH, Xu D, Yuan SX, Zhu N, Zhou WP, Yang GS, Wang YZ, et al: Long noncoding RNA high expression in hepatocellular carcinoma facilitates tumor growth through enhancer of zeste homolog 2 in humans. Hepatology 54: 1679-1689, 2011.

15. Enfield KS, Pikor LA, Martinez VD and Lam WL: Mechanistic roles of noncoding RNAs in lung cancer biology and their clinical implications. Genet Res Int 2012: 737416, 2012.

16. Chai Y, Liu J, Zhang Z and Liu L: HuR-regulated lncRNA NEAT1 stability in tumorigenesis and progression of ovarian cancer. Cancer Med 5: 1588-1598, 2016.

17. Qi P, Dong L, Lin W, Zhou X and Du X: Abstract B42: A two-lncRNA signature in serous exosomes serves as a new biomarker for colorectal cancer diagnosis. Cancer Res 76: B42, 2016.

18. Piao HL and Ma L: Non-coding RNAs as regulators of mammary development and breast cancer. J Mammary Gland Biol Neoplasia 17: 33-42, 2012.

19. Zhao W, An Y, Liang Y and Xie XW: Role of HOTAIR long noncoding RNA in metastatic progression of lung cancer. Eur Rev Med Pharmacol Sci 18: 1930-1936, 2014.

20. Mercer TR, Dinger ME and Mattick JS: Long non-coding RNAs: Insights into functions. Nat Rev Genet 10: 155-159, 2009.

21. Gao J, Cao R and Mu H: Long non-coding RNA UCA1 may be a novel diagnostic and predictive biomarker in plasma for early gastric cancer. Int J Clin Exp Pathol 8: 12936-12942, 2015.
22. Jiang L, Lai YK, Zhang J, Wang H, Lin MC, He ML and Kung HF Targeting S100P inhibits colon cancer growth and metastasis by Lentivirus-mediated RNA interference and proteomic analysis. Mol Med 17: 709-716, 2011.

23. Livak KJ and Schmittgen TD: Analysis of relative gene expression data using real-time quantitative PCR and the 2(-Delta Delta $\mathrm{C}(\mathrm{T})$ ) method. Methods 25: 402-408, 2001

24. Bierhoff H, Schmitz K, Maass F, Ye J and Grummt I: Noncoding transcripts in sense and antisense orientation regulate the epigenetic state of ribosomal RNA genes. Cold Spring Harb Symp Quant Biol 75: 357-364, 2010.

25. Wang KC and Chang HY: Molecular mechanisms of long noncoding RNAs. Mol Cell 43: 904-914, 2011.

26. Nagano T and Fraser P: No-nonsense functions for long noncoding RNAs. Cell 145: 178-181, 2011.

27. Loewer S, Cabili MN, Guttman M, Loh YH, Thomas K, Park IH, Garber M, Curran M, Onder T, Agarwal S, et al: Large intergenic non-coding RNA-RoR modulates reprogramming of human induced pluripotent stem cells. Nat Genet 42: 1113-1117, 2010.

28. Saxena A and Carninci P: Long non-coding RNA modifies chromatin: Epigenetic silencing by long non-coding RNAs. Bioessays 33: 830-839, 2011.

29. Kotake Y,Nakagawa T, Kitagawa K, Suzuki S, Liu N, Kitagawa M and Xiong Y: Long non-coding RNA ANRIL is required for the PRC2 recruitment to and silencing of p15(INK4B) tumor suppressor gene. Oncogene 30: 1956-1962, 2011.

30. Tsai MC, Manor O, Wan Y, Mosammaparast N, Wang JK, Lan F, Shi Y, Segal E and Chang HY: Long noncoding RNA as modular scaffold of histone modification complexes. Science 329: 689-693, 2010.

31. Noori-Daloii MR and Eshaghkhani Y: lncRNAs roles in cancer occurrence. Med Sci J Islamic Azad Univesity-Tehran Medical Branch 25: 163-182, 2015.

32. Lin A, Li C, Xing Z, Hu Q, Liang K, Han L, Wang C, Hawke DH, Wang S, Zhang Y, et al: The LINK-A lncRNA activates normoxic HIF1 $\alpha$ signalling in triple-negative breast cancer. Nat Cell Biol 18: 213-224, 2016.

33. Xu Z, Chen J, Luk JM and De W: Abstract 157: LncRNA ANRIL indicates a potential prognostic biomarker in gastric cancer and promotes tumor growth by silencing of miR-99a/miR-449a. Cancer Res 75: 157, 2015.

34. Chen T, Xie W, Xie L, Sun Y, Zhang Y, Shen Z, Sha N, Xu H, $\mathrm{Wu} \mathrm{Z}, \mathrm{Hu} \mathrm{H}$ and Wu C: Expression of long noncoding RNA lncRNA-n336928 is correlated with tumor stage and grade and overall survival in bladder cancer. Biochem Biophys Res Commun 468: 666-670, 2015.

35. Zhang G, Hou X, Li Y and Zhao M: MiR-205 inhibits cell apoptosis by targeting phosphatase and tensin homolog deleted on chromosome ten in endometrial cancer Ishikawa cells. BMC cancer 14: 440, 2014.

36. Yang F, Bi J, Xue X, Zheng L, Zhi K, Hua J and Fang G: Up-regulated long non-coding RNA H19 contributes to proliferation of gastric cancer cells. FEBS J 279: 3159-3165, 2012.

37. Yarmishyn AA and Kurochkin IV: Long noncoding RNAs: A potential novel class of cancer biomarkers. Front Genet 6: 145 , 2015.

38. Endo H, Shiroki T, Nakagawa T, Yokoyama M, Tamai K, Yamanami H, Fujiya T, Sato I, Yamaguchi K, Tanaka N, et al: Enhanced expression of long non-coding RNA HOTAIR is associated with the development of gastric cancer. PLoS One 8: e77070, 2013.

39. Xu ZY, Yu QM, Du YA, Yang LT, Dong RZ, Huang L, Yu PF and Cheng XD: Knockdown of long non-coding RNA HOTAIR suppresses tumor invasion and reverses epithelial-mesenchymal transition in gastric cancer. Int J Biol Sci 9: 587-597, 2013

40. Sun M, Jin FY, Xia R, Kong R, Li JH, Xu TP, Liu YW, Zhang EB, Liu XH and De W: Decreased expression of long noncoding RNA GAS5 indicates a poor prognosis and promotes cell proliferation in gastric cancer. BMC Cancer 14: 319, 2014.

41. Wang J, Su L, Chen X, Li P, Cai Q, Yu B, Liu B, Wu W and Zhu Z: MALAT1 promotes cell proliferation in gastric cancer by recruiting SF2/ASF. Biomed Pharmacother 68: 557-564, 2014. 